Recepción: 05/01/2019

Aceptación: 11/03/2019

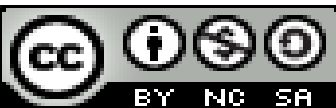

Ciencias económicas y empresariales

Artículo de revisión

\title{
La oferta exportable de las PYMES de la provincia de Manabí
}

\section{The exportable offer of the PYMES of the province of Manabi A oferta exportável do PYMES da província de Manabí}

\author{
Guadalupe María Leonor Toala-Toala ${ }^{\mathrm{I}}$ \\ gtoala2000@hotmail.com \\ Amado Antonio Mendoza-Briones II \\ antoniomendoza1971@yahoo.com \\ Dellaneira Lisbeth Vinces-Menoscal ${ }^{\text {III }}$ \\ dellalis@gmail.com \\ Luís M. Moreira-Moreira ${ }^{\mathrm{IV}}$ \\ mmoreira.moreira3@gmail.com
}

Correspondencia: gtoala2000@hotmail.com

I Magíster en Gerencia de Proyectos Educativos y Sociales, Diploma Superior en Educación Universitaria por Competencias, Doctora en Ciencias Administrativas, Especialista en Diseño Curricular por Competencia, Ingeniera Comercial, Docente Universidad Laica "Eloy Alfaro” de Manabí, Manta, Ecuador.

II Magíster en Gerencia de Proyectos Educativos y Sociales, Diploma Superior en Educación Universitaria por Competencias, Doctor en Ciencias Administrativas, Licenciado en Ciencias de la Educación Especialidad Físico Matemáticas, Profesor de Segunda Enseñanza Especialidad Físico Matemáticas, Docente Universidad Laica "Eloy Alfaro" de Manabí, Manta, Ecuador.

Estudiante de la Universidad Laica “Eloy Alfaro” de Manabí, Manta, Ecuador. 


\title{
Resumen
}

El presente trabajo de investigación se realiza con el objetivo de conocer el motivo por el cual, las pequeñas y medianas empresas de Manabí no logran, en muchas ocasiones, expandirse a mercados nacionales o mercados internacionales. Las pequeñas y medianas empresas se han convertido, en la actualidad en el eje transversal del crecimiento de los países y sus economías indistintamente de los niveles de desarrollo de cada uno, en donde, el Gobierno Provincial de Manabí está buscando e ideando estrategias que le permitan expandir dicha oferta exportable de la provincia con la finalidad de hacer crecer la entrada de divisas. El artículo consta del análisis del planteamiento del problema relacionado con la oferta exportable de las PYMES, objetivos a tener en cuenta y a desarrollarse por medio de la elaboración del proyecto, en donde se analizarán factores que inciden dentro de la investigación, para determinar en sí cuáles de estos factores influyen de manera directa o indirecta en la oportunidad de exportación de las Pymes. La metodología utilizada se trata de una investigación bibliográfica-documental.

Palabras clave: Oferta exportable; Pymes; comercio internacional; exportación.

\begin{abstract}
The present research work is carried out with the objective of knowing the reason why, the small and medium companies of Manabí do not manage, in many occasions, to expand to national markets or international markets. Small and medium-sized enterprises have become, at present, the transversal axis of the growth of the countries and their economies regardless of the levels of development of each, where, the Provincial Government of Manabí is looking for and devising strategies that allow it expand said exportable offer of the province in order to increase the entrance of foreign currency. The article consists of the analysis of the approach to the problem related to the exportable supply of SMEs, objectives to be taken into account and developed through the elaboration of the project, where factors that affect the research will be analyzed, in order to determine which ones of these factors directly or indirectly influence the export opportunity of SMEs. The methodology used is a bibliographic-documentary investigation.
\end{abstract}

Keywords: Exportable offer; SMEs; international trade; Exportation. 


\section{Resumo}

O presente trabalho de pesquisa é realizado com o objetivo de saber o porquê, as pequenas e médias empresas de Manabí não conseguem, em muitas ocasiões, se expandir para mercados nacionais ou mercados internacionais. Pequenas e médias empresas tornaram-se, agora em eixo secundário do crescimento dos países e suas economias, independentemente dos níveis de esenvolvimento de cada um, onde o Governo da Província de Manabí está buscando e criando estratégias para você expandir a referida oferta exportável da província, a fim de aumentar a entrada de moeda estrangeira. $\mathrm{O}$ artigo consiste em abordagem de análise de problemas relacionados com a oferta de exportação das PME, tem como objetivo analisar e desenvolver através do desenvolvimento do projeto, onde os fatores envolvidos na investigação serão analisados para determinar-se o desses fatores influenciam direta ou indiretamente a oportunidade de exportação das PMEs. A metodologia utilizada é uma investigação bibliográfico-documental. Palavras-chave: Oferta exportável; PME; comércio internacional; exportação.

\section{Introducción}

Dentro de un mundo globalizado la competitividad en el mundo de las exportaciones, ayuda a un país u organización a permanecer en constante evolución y a mejorar, por ende, sus ingresos. Cuando hablamos de oferta exportable, nos referimos a la capacidad que tiene un país o empresa de ofrecer un producto nacional a los mercados internacionales, por lo cual se requiere como condición necesaria, contar con un producto o servicio que cumpla con todos los estándares internacionales de calidad y satisfacción al cliente, para que este pueda competir en dichos mercados.

La capacidad de ofrecer un producto o servicio que sea acorde a lo que el cliente desea, también se enfoca en lo que la empresa pueda ofrecer y esté en condiciones de hacerlo. Una vez que cuente con el producto, deberá analizar diversas preguntas, como esta ¿Es la exportación la mejor opción o lo es el mercado nacional?, en donde se analiza la opción de comerciar internacionalmente, especialmente si se va a iniciar. Según un reporte publicado en enero (2018) por el Centro del Comercio Internacional, a más de 700 entrevistados que cubren los principales sectores de exportación, en cada cinco exportadores, mencionan que existen dificultades ligadas a las medidas arancelarias, tres de cuatro dificultades están ligadas a reglamentaciones aplicadas 
por los países de destinos y la última cuarta parte, está ligada a las medidas ecuatorianas aplicadas a las exportaciones.

En un artículo publicado por Pro-Ecuador, se hace referencia a que el país participa "continuamente en ferias internacionales, misiones comerciales, ruedas de negocios y eventos de promoción comercial que permiten promover los negocios entre Ecuador y el mundo". Ecuador como un país exportador, está ligado a que sus empresas exporten para aumentar el ingreso del mismo, por lo cual el país debe incentivar y comprometerse con las empresas, incluidas las Pymes, en impulsar y mejorar la calidad de sus exportaciones.

En Ecuador, las Pymes no se ven tan ligadas a dichas exportaciones a nivel internacional, debido a un innumerable número de factores, sean tecnológicos o financieros. Dentro del Ecuador, Manabí cuenta con un total de 63.690 empresas, de las cuales, el 99.7\% son micro, medianas y pequeñas empresas. Los cantones de Portoviejo y Manta concentran el mayor número de empresas de la provincia, que se dedican principalmente al comercio de un $37 \%$ del total del país, el $34 \%$ a los servicios, el $18 \%$ a la agricultura, el $8 \%$ a la industria manufacturera y el $3 \%$ construcción. (INEC, 2016).

El problema de exportación en Manabí, radica, en que el estado actual de las PYMES, impide que estas comercialicen por encima de un nivel exportable, en donde factores como: un escaso nivel tecnológico, baja calidad de la producción, ausencia de normas y altos costos, falta de crédito, difícil acceso, mano de obra sin calificación, producción orientada a niveles locales y mercado interno, entre otros, incluidas las políticas y estrategias para desarrollar dentro del sector, son impedimentos para una expansión internacional o nacional.

En este contexto, se imbrica este proyecto, con el cual se busca diagnosticar la oferta exportable de las Pymes de la provincia de Manabí, con el propósito de consolidar e impulsar el comercio internacional entre dichas empresas y el mercado internacional, por lo cual, los representantes de estas medianas y pequeñas empresas, deben fortalecer la competitividad y productividad mediante el acceso a créditos, diseñar programas, políticas y estrategias que le permitan comercializar a niveles nacionales en primer lugar, que estén enfocadas a un comercio exterior 
futuro y a una implementación de nuevas tecnologías y capacitación de los recursos humanos, con el fin de promover el incremento y diversificación de la oferta exportable.

En ese orden la presente investigación se justifica en la necesidad que tiene el país, y la provincia de Manabí, de impulsar la oferta exportable de sus pequeñas y medianas empresas para de esta manera poder genera divisas, como un medio de sustentabilidad para la economía ecuatoriana, principalmente para la provincia de Manabí, la cual siempre fue una contribuyente real y efectiva a la seguridad alimentaria del país, las principales actividades de sus habitantes siempre fueron la agropecuaria y la pesca, ambas fundamentales para la subsistencia y dieta diaria de la población provincial y nacional, fue muy fecunda en el abastecimiento de productos de consumo nacional, pero también fue un baluarte en el comercio exterior y exportaciones del país. (El Diario, 2015). Manabí, se clasifica como una provincia exportadora de diferentes productos, tales como: café, cacao, atún, plátano y camarón, ha logrado exportar dichos productos a un mercado internacional, en donde, el cacao ha sido y es uno de los productos exportables con gran producción en el país.

Por esas razones mediante esta investigación, se busca incentivar a las Pymes de la provincia, a tomar acciones que los ayuden a internacionalizarse buscando mejores ingresos y a su vez mejorando la economía de la provincia y del país, ya que una de las ventajas que poseen las Pymes, frente a las grandes empresas, es que cuentan con una gran y rápida adaptación a los requerimientos del mercado debido a que poseen estructuras más pequeñas. Además, de ser considerado el sector con mayor generador de empleo y poseedor de gran reacción al cambio.

\section{Materiales y Métodos}

\section{¿Qué es el Comercio Internacional?}

El comercio internacional estudia al conjunto de movimientos o transacciones comerciales y financieros que se realizan entre dos o más países y su objetivo es lograr entre todos los países un crecimiento armónico y equilibrado (Krugman, 2000). Un país defiende su Producto Interno Bruto (PIB) y aplica su propia política económica (Política cambiaria TC, Política Fiscal, Política Monetaria, Política de comercio exterior, etc.) Para controlar y dirigir su economía hacia los objetivos macroeconómicos de bienestar social. Así lo que haga un país en materia de política económica con seguridad afecta a otros países; por ejemplo, el incremento en los aranceles para 
defender la competitividad nacional y masificar las ventas al exterior (exportaciones) afecta a los países desarrollados tradicionalmente exportadores.

En ese contexto cabe referir que, el comercio internacional estudia la Balanza Comercial (BC)y la Balanza de Pagos (BP), que se expresan a través de las exportaciones, las importaciones, la entrada y salida de activos financieros, el resultado del comercio internacional mucho depende de las políticas económicas adoptadas por cada país. (Guerra, 2012).

\section{Oferta Exportable}

La autora Rozo Omaira (2014), refiere que "la oferta exportable se puede definir como los productos que se encuentran en condiciones de exportación dentro de un país, ya que al ser elaborados a mayor volumen están en capacidad de cubrir la demanda nacional e internacional (según sea necesario) de forma estable y continúa." (p. 35), lo cual tiene el propósito de desarrollar acciones orientadas a lograr oferta estratégicamente diversificada, con significativo valor agregado de calidad y volumen, que permitan tener una presente competencia en los mercados internacionales. La oferta exportable de una empresa, es más que asegurar los volúmenes solicitados por un determinado cliente o contar con productos que satisfacen los requerimientos de los mercados de destino, según Irene Cerón, "La expansión de las exportaciones estará condicionada a la ampliación y diversificación de mercados, remoción de medidas proteccionistas en los mercados de destino, mejoramiento de calidad y de sistemas de producción, transporte, manejo y comercialización, y diversificación de la producción exportable." (P. 39)

Aunado a lo anterior, cabe acotar que, la oferta exportable también tiene que ver con la capacidad económica, financiera y de gestión de la empresa como, por ejemplo:

1. Disponibilidad del producto.

2. Capacidad de Gestión.

3. Capacidad de Producción.

4. Capacidad de especialización. 


\section{Disponibilidad del Producto}

Con base en el producto que desea exportar, la empresa debe determinar el volumen que tiene disponible para el mercado externo. El volumen debe ser aquel que pueda ofrecer de manera estable o continua. De igual modo, el producto debe cumplir con las exigencias y requerimientos del mercado objetivo. Para las empresas es necesario entregar una buena experiencia de compra ofreciendo productos y servicios adecuados a las necesidades de los consumidores dada la alta competencia existente. Por esta razón es importante cuantificar y estudiar el efecto que los problemas en la disponibilidad de los productos generan sobre los clientes. (Von Hausen Jacqueline, 2014).

\section{Capacidad de Gestión}

Gestionar quiere decir realizar las acciones que debemos llevar adelante para resolver nuestros problemas, para satisfacer las necesidades y demandas que nos propusimos encarar como organización. La empresa debe desarrollar una cultura exportadora, con objetivos claros y conocimiento de sus capacidades y debilidades para afrontar a las diversas actividades que significan encaminarse a una producción y exportación, sean estas nacionales o internacionales.

La organización tiene su modo de actuar y decide lo que va a hacer para resolver sus problemas y satisfacer sus necesidades. Claro que muchas veces lo que hace una organización va en contra o afecta lo que pretende hacer otra, porque para satisfacer sus necesidades un grupo o sector afecta a otros, por lo cual, como organización se debe tener iniciativas propias para buscar soluciones a los problemas y decidir las acciones a realizar sin depender de otros, entonces la empresa se está auto gestionando. (CENOC, 1999).

\section{Capacidad de Producción}

La capacidad es definida como el volumen de producción recibido, almacenado o producido sobre una unidad de tiempo, siendo producción el bien que produce la empresa, ya sea intangible o no. Una empresa puede planear su capacidad a largo, mediano o corto plazo, con el fin de garantizar una producción enfocada a la estrategia de competitividad de la empresa, modificando instalaciones, mano de obra y maquinaria. 
La capacidad de producción determina factores como tiempos, unidades, recursos que serán utilizados en la transformación de materiales u objetos en un periodo de tiempo determinado, teniendo en cuenta la demanda del mercado, la suficiencia y la disponibilidad de los recursos físicos e intangibles de la empresa, una capacidad excesiva con una baja demanda, tendrá elevados costos en el funcionamiento de la planta, mientras que una capacidad que no consigue igualar el nivel de demanda, resulta insuficiente conllevando a la pérdida de competitividad.

\section{Capacidad de Especialización}

La no neutralidad de las características de la estructura sectorial, en relación con los efectos multiplicadores que tendrían las exportaciones sobre el tejido productivo marca la relevancia de estudiar el perfil de especialización comercial de los países. El estudio del patrón de especialización internacional ha sido abordado por distintas escuelas teóricas, interesadas tanto por cuestiones analíticas como por las controversiales derivaciones de política que pueden desprenderse de su análisis. (Stumpo Giovanni, CEPAL, 2009).

Según el referido autor Stumpo aporta que: "el enfoque evolucionista concluye que el patrón de especialización adquiere una fuerte relevancia sobre el sendero de crecimiento de un país". (p. 25) Esto parece confirmarse a partir de la experiencia de los países exitosos de industrialización reciente como Corea, Taiwán, Malasia y Tailandia, que muestran, cambios importantes en sus patrones de especialización, a favor de sectores de mayor nivel tecnológico, mayor intensidad en el uso de la mano de obra calificada y con una demanda internacional más dinámica.

\section{La competitividad y el comercio exterior}

Un país se considera competitivo si su balanza comercial es superavitaria. El indicador total de competitividad es un promedio ponderado de la capacidad de importación y exportación de un país y se supone que refleja la posición de éste en ambos mercados: el nacional y el extranjero. $\mathrm{Si}$ el objetivo es la evaluación del desempeño macroeconómico del país, entonces no sólo se deberían enfocar los factores de precio e ignorar otros de carácter estructural, como la capacidad para la innovación tecnológica, el grado de especialización y la calidad de los productos involucrados, la calidad del sistema de educación, infraestructura y salud pública, así como el 
sistema político y el tipo de políticas macroeconómicas, entre otras. (Bougrine, H., 2001, Competitividad y comercio exterior. Comercio Exterior).

\section{Las Nuevas Teorías del Comercio Internacional}

Ante los importantes cambios en la competencia que comenzaban a darse en la década de los setenta $\mathrm{y}$, por tanto, ante la irrealidad de algunos de los supuestos de partida de la teoría neoclásica del comercio internacional, comienzan en esta época, a elaborarse algunas teorías explicativas del comercio internacional que partían de la flexibilización de algunos de los supuestos, anteriormente comentados, de la teoría neoclásica. Dichas teorías se encuadran dentro del grupo denominado "nuevas teorías del comercio internacional” (NTCI). (Flores, 2008) Estas teorías no son más que una continuación de las teorías neoclásicas, pues, aunque parten de los mismos supuestos, sustituyen el de competencia perfecta por el de competencia imperfecta, siguiendo a Guerrero (1995), sobre estas teorías caben realizar las siguientes afirmaciones:

- No se trata de teorías opuestas, sino que son una continuación de las teorías neoclásicas, pues tratan de complementarse y explicar diferentes aspectos de la realidad del comercio internacional.

- Cuando se realiza un análisis profundo de las mismas, algunos supuestos de partida, en principio diferentes, tienden a desaparecer, por lo que ambas convergen a un mismo enfoque, es decir, a una misma filosofía de entender la competencia.

Ambos grupos de teorías parten de un mismo paradigma y comparten una serie de limitaciones. En este sentido, puede decirse que la existencia de una única función de producción en cada sector productivo es el supuesto común, y este sí es un supuesto esencial, en la cuales se le da mucha importancia a la productividad del trabajo y al factor trabajo, como parte esencial de ésta. 


\section{Organización industrial y comercio internacional}

Estas teorías tratan de relacionar la organización industrial de un país con el comercio internacional. En este sentido, analizan aspectos tales como la estructura de la demanda interna como prerrequisito para la exportación, la influencia de la estructura de los mercados interiores sobre los flujos del comercio internacional, el incremento del comercio internacional sobre la eficiencia técnica y las economías de escala o la interrelación entre el comercio internacional y diferenciación del producto. En definitiva, estos modelos pretenden explicar el comercio internacional de los países en función de las estructuras internas de sus mercados e industrias. (Flores, 2008).

\section{Economías de escala}

Las teorías pertenecientes a este modelo pasan, de suponer rendimientos constantes, tal y como lo hacen las teorías neoclásicas de competencia perfecta, a suponer economías de escala, supuesto típico de mercados con competencia imperfecta. Estas teorías no pretenden justificar el comercio internacional en función de las diferencias factoriales entre países, las cuales explican el comercio interindustrial en la teoría neoclásica, sino que, son los rendimientos crecientes, los que pueden explicar, en buena parte, la distribución geográfica de la actividad económica.

No obstante, autores como Guerrero (1995) afirman que estas teorías siguen manteniendo, al igual que la teoría neoclásica, el supuesto de la misma función de producción para todas las empresas de un sector. Al mismo tiempo, señalan que las economías de escala no justifican por sí solas el comercio internacional, siendo necesario recurrir, en última instancia, a la ventaja comparativa. Y, por último, también se debe resaltar que estas teorías son incapaces de prever cuáles serán las empresas y países que aprovechen las economías de escala o, al menos, determinar en qué proporción lo harán. 


\section{Resultados y Discusión}

\section{Diferenciación de producto y comercio intraindustrial}

Estas teorías intentan explicar las razones que impulsan el incremento del comercio intraindustrial que se ha venido experimentando en las últimas décadas. En este sentido, existen diversos trabajos que apuntan a la progresiva diferenciación de los productos como uno de los principales factores desencadenantes de este proceso. Por tanto, en esta teoría, la empresa pasa a jugar un papel más destacado en la competitividad del país, pues esta competitividad, en este caso, no sólo viene determinada por la estructura de costes, sino que factores como la calidad, el diseño o las distintas prestaciones de los productos pasan a jugar un papel importante en el comercio internacional, sobre todo para bienes homogéneos, es decir, para el comercio intraindustrial.

Las nuevas teorías del comercio internacional, tan sólo representa una continuación de las teorías neoclásicas del comercio internacional, ya que suponen la introducción de nuevos supuestos que permiten adaptarse a los cambios que, a partir de los sesenta, comienzan a experimentarse en la economía internacional, competencia imperfecta, competencia monopolística, diferenciación de productos, economías de escalas, etc. (Flores, 2008).

No obstante, conviene decir que subsisten todavía numerosas lagunas y puntos oscuros, en especial, por lo que respecta a la inclusión de los factores dinámicos relacionados con el cambio técnico y sus efectos sobre la evolución temporal de los patrones de especialización (Bajo 1991).

\section{Características y Aporte de las Pymes}

Aunado a los aspectos anteriores, es relevante referir que, las empresas en Ecuador están clasificadas de acuerdo a su tamaño, tienen las siguientes categorías:

- Microempresas: emplean hasta 10 trabajadores, y su capital fijo (descontado edificios y terrenos) puede ir hasta 20 mil dólares.

- Talleres artesanales: se caracterizan por tener una labor manual, con no más de 20 operarios y un capital fijo de 27 mil dólares. 
- Pequeña Industria: puede tener hasta 50 obreros

- Mediana Industria: alberga de 50 a 99 obreros, y el capital fijo no debe sobrepasar de 120 mil dólares.

- Grandes Empresas: son aquellas que tienen más de 100 trabajadores y 120 mil dólares en activos fijos

En la siguiente tabla se expone el número de empresas por sector y su participación en la generación de empleos, tal como se expresa a continuación:

Tabla $\mathbf{N}^{0}$ 1. Empresas por sector y su participación en la generación de empleo,

\begin{tabular}{lccc}
\hline \multicolumn{1}{c}{ SECTOR } & $\begin{array}{c}\text { NUMERO } \\
\text { EMPRESAS }\end{array}$ & $\begin{array}{c}\text { PROMEDIO EMPLEADOS } \\
\text { POR EMPRESA }\end{array}$ & TOTAL \\
& 15.000 & 22 & 330.000 \\
\hline PYMIS & 200.000 & 3 & 600.000 \\
Artesanías & 252.000 & 3 & 756.000 \\
Microempresas & $\mathbf{4 6 7 . 0 0 0}$ & & $\mathbf{1 ' 6 8 6 . 0 0 0}$ \\
\hline TOTAL & & &
\end{tabular}

Fuente: Revisión documental bibliográfica. Elaboración propia. (2018).

\section{Conclusiones}

El estudio de la oferta exportable, los negocios universales en el comercio internacional ratifican los procesos actuales que son de enorme importancia, pues es posible registrar y clasificar de manera ordenada la información que se desea conocer, donde la praxis retroalimenta los hallazgos empíricos con su quehacer teórico. Sumado a lo anterior, desde un punto de vista de investigación cuantitativa, se avanza hacia la construcción de bases de datos que dan cuenta de los procesos y de la información detallada en un orden coherente y el impacto que estos espacios han tenido en determinados contextos; y desde un punto de vista cualitativo, es posible observar características, factores o rasgos distintivos del fenómeno que visibilizan la homogeneidad o heterogeneidad de estos campos en cada ambiente de análisis. 
En consecuencia, este estudio contribuye a la evidencia empírica asociada a comprobar la relación entre la oferta exportable, las barreras a la exportación y su influencia en el compromiso exportador y sus repercusiones en los resultados internacionales de las Pymes en Ecuador.

Los temas relacionados con el mundo del comercio internacional y los negocios internacionales abordados en este documento permiten delimitar lugares de trabajo que se puede estudiar sistemáticamente. Ciertas aristas de investigación pueden ser encaminadas hacia temas específicos y áreas problemáticas que conlleven la realización de proyectos de investigación, que interdisciplinariamente y críticamente se enfoquen en el comercio internacional e internacional, así como las relaciones de negociación derivados de este. Si bien el abanico de posibilidades es amplio, se propone como ejes temáticos los siguientes:

1. Determinantes del comercio internacional (desempeño exportador e importador) y de inversión extranjera en distintos niveles: micro, meso, macro y regional;

2. Volatilidad cambiaria y afectación del comportamiento competitivo empresarial y sectorial;

3. Internacionalización empresarial y capacidad exportadora;

4. Medición de la apertura de mercados nacional, departamental y sectorial, y

5. Aprovechamiento

\section{Referencias Bibliográficas}

ÁNGEL ÁLVAREZ, B. E., 2014. El concepto de Innovación. Bogotá-Colombia.

ARAQUE JARAMILLO, W., 2015. Potencial de Exportación de las Pymes. Revista Gestión, Economía y Sociedad.

ARNOLDO ARAYA, L., 2009. El proceso de Internacionalización de las Empresas. Dialnet. TEC Empresarial Ed.3. 
ASTUDILlO MOYA, M., 2013. Fundamentos de Economía. México: UNAM: Instituto de Investigaciones Económicas: Probooks.

BETANCOURT, D., 2016. Ingenio Empresa. Obtenido de https://ingenioempresa.com/capacidad-produccion-empresa/

CANO FLORES, M., OLIVERA GOMEZ, D. A., \& HERNÁNDEZ OLVERA, A., 2014. La comercialización Internacional: Alternativa de Crecimiento de las Pyme. R. Ciencia Administrativa No.1.

CARVACHE FRANCO, O., 2017. La Cultura Organizacional para la innovación y el desarrollo competitivo de las pequeñas y medianas empresas (PYMES) exportadoras del Guayas. Revista Dialnet.

CENTRO DE COMERCIO INTERNACIONAL. 2018. Ecuador: Perspectivas empresariales Una serie del ITC sobre las medidas no arancelarias. Ginebra.

COELLO CAZAR, D., 2016. Caracterización de pequeñas y medianas empresas exportadoras. Un estudio exploratorio para el caso ecuatoriano. Revista Dialnet.

CONCEJO NACIONAL DE COORDINACIÓN. 2010. Capacitación de organizaciones de base. Paquete Audiovisual Didáctico.

DE LA HOZ CORREA, P. A., 2014. Generalidades de Comercio Internacional. Medellín: Centro Editorial Esume.

Delgado, D. D., \& CHÁveZ GRANizO, G. P., 2018. Las Pymes y su fuente de Financiamiento. Revista Observatorio de la Economía Latinoamericana. Obtenido de https://www.eumed.net/rev/oel/2018/04/pymes-ecuador-financiamiento.html

El Diario. (Julio de 2011). Cinco productos manabitas generan más divisas al país (Manabí). El Diario Ec.

El Diario. (Julio de 2015). Plátano, café y cacao, lo más exportado (Manabí). El Diario. Ec. 
ESCANDÓN BARBOSA, D. M., \& HURTADO AYALA, A., 2014. Los determinantes de la orientación exportadora y los resultados en las pymes exportadoras en Colombia. sciencedirec.

ESPINOSA AYALA, E., 2010. La competitividad del Sistema Agroalimentario localizado productos de Quesos Tradicionales. Toluca, Estado de México.

FANJUL, E., 2017. Iber global. Obtenido de www.iberglobal.com: http://www.iberglobal.com/files/2017/que_es_internacionalizacion-B.pdf

FLORES, R. D., 2008. Competitividad Sostenible de los espacios naturales protegidos como destinos turísticos: Un análisis comparativo de los parques naturales Sierra de Aracena y Picos de Aroche y Sierras de Cazorla, Segura y las Villas. España: Tesis doctoral.

FROHMANN, A., MULDER, N., OLMOS, X., \& URMENETA, R., 2016. Internacionalización de las Pymes. Innovación para exportar. Santiago: Publicación de las Naciones Unidas (CEPAL).

GARCIA DEL JUNCO, J., CASANUEVA ROCHA, C., GANAZA VARGAS, J., \& ALONSO RODRIGUEZ, M. Á., 2000. Prácticas de la Gestión Empresarial. España: Mc.Graw-Hill Interamericana de España.

GARCIA, E., 2013. Comercialización internacional. Buenos Aires: Editorial Estudiantil S.A

GOMEZ-DÍAZ, C., \& RODRÍGUEZ ORTIZ, J. K. (2015). Teorías de la Cultura Organizacional. ResearchGate.

GUERRERO, D., 1995. Competitividad: Teória y política. Ariel: Barcelona.

IAVARONE, P. G., 2012. Costos por órdenes de producción: su aplicación a la industria panificadora. Provincia de Mendoza - Argentina.

IRENE CERÓN R., 2016. Oferta Exportable de Productos Agrícolas. Universidad de Chile. 
MINISTERIO DE AGRICULTURA Y RIEGO. (2009). Minagri-Perú. Obtenido de http://minagri.gob.pe/portal/objetivos/181-exportaciones/que-podemos-exportar/532definicion-de-oferta-exportable

MOUSALLI-KAYAT, G., 2015. Métodos y Diseños de Investigación Cuantitativa. Revista researchgate. Obtenido de https://www.researchgate.net/publication/303895876_Metodos_y_Disenos_de_Investiga cion_Cuantitativa

MULDER, N., \& PELLANDRA, A. (2017). La innovación exportadora en las pequeñas y medianas empresas. Programas de apoyo y financiamiento en América Látina. Santiago: Publicación de las Naciones Unidas. (CEPAL).

ORGANIZACIÓN MUNDIAL DEL COMERCIO., 2005. Informe sobre el Comercio Mundial. Análisis sobre los vínculos entre el comercio, las normas y la OMC.

OTZEN, T., \& MANTEROLA, C., 2017. Técnicas de Muestreo sobre una Población a Estudio. Int. J. Morphol.

PASCUAL, R. F., \& GHIOTTO, L., 2010. Re conceptualizando lo político: Estado, mercado mundial, globalización y neoliberalismo. scielo.org. 60

PRO-ECUADOR (21 de mayo del 2018) www.proecuador.gob.ec Obtenido en: https:/www.proecuador.gob.ec/oferta-exportable-ecuatoriana-destaca-en-corea-del-sur/

RESTREPO MORALES, J. A. (2015). Aplicación de un método multicriterio, basado en lógica difusa, para medir capacidad exportadora: Validación en el clúster textil/confección de la ciudad de Medellín. Revista Research Gate.

RIVERA RODRIGUEZ, H. A., \& MALAVER ROJAS, M. N., 2011. ¿Qué estudia la estrategia? Bogotá: Editorial Universidad del Rosario.

ROZOR, O. (2014). En que consiste la Oferta Exportable. Obtenido de Asesorías Fijas ANFER: http://omairarozor.blogspot.com/2014/04/en-que-consiste-la-oferta-exportable.html 
SUBÍA VELOZ, R. C., BARZAGA SABLÓN, O. S., \& NEVARES BARBERÁN, V., 2018. El financiamiento de las medianas empresas en la provincia Manabí, Ecuador. Revista Trimestral Ciencias Holguín Vol.24 No.1.

VALENCIA, A., 2013. ENTREVISTA-Ecuador quiere ampliar oferta exportable para disminuir dependencia de petróleo. Agencia de Noticias Reuters.

VALERO CÓRDOBA, G. M., RODENES, M., \& ELENA RUEDA, G., 2016. La internacionalización de las empresas exportadoras. Estado de la Cuestión. Revista Lebret.

VALERO CÓRDOBA, G., RODENES, M., \& ELENA RUEDA, G., 2016. Influencia de la innovación en la competitividad de las empresas exportadoras - estado de la cuestión. Memoria del X Congreso de la Red Internacional de Investigadores en Competitividad.

VON HAUSEN, J., 2014. Efecto de Disponibilidad de Variedad de productos en el comportamiento de clientes. Santiago de Chile. 